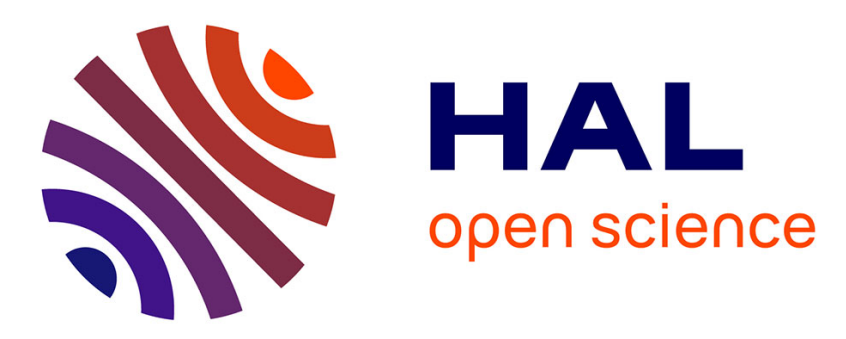

\title{
A new energy detector of micro-emboli using a time-varying threshold
}

\author{
Maroun Geryes, Sébastien Ménigot, Walid Hassan, Marilys Almar, Benoit \\ Guibert, Corinne Gautier, Jamal Charara, Jean-Marc Girault
}

\section{To cite this version:}

Maroun Geryes, Sébastien Ménigot, Walid Hassan, Marilys Almar, Benoit Guibert, et al.. A new energy detector of micro-emboli using a time-varying threshold. 2015 International Conference on Advances in Biomedical Engineering (ICABME), Sep 2015, Beyrouth, Lebanon. pp.89-92, 10.1109/ICABME.2015.7323258 . hal-01279950

\section{HAL Id: hal-01279950 https://hal.science/hal-01279950}

Submitted on 28 Feb 2016

HAL is a multi-disciplinary open access archive for the deposit and dissemination of scientific research documents, whether they are published or not. The documents may come from teaching and research institutions in France or abroad, or from public or private research centers.
L'archive ouverte pluridisciplinaire HAL, est destinée au dépôt et à la diffusion de documents scientifiques de niveau recherche, publiés ou non, émanant des établissements d'enseignement et de recherche français ou étrangers, des laboratoires publics ou privés. 


\title{
A New Energy Detector of Micro-Emboli Using a Time-Varying Threshold
}

\author{
Maroun GERYES ${ }^{1,2}$, Sébastien MENIGOT ${ }^{1}$, Walid HASSAN ${ }^{2}$, Marilys ALMAR ${ }^{3}$, Benoit Guibert ${ }^{3}$, Corinne \\ GAUTIER4, Jamal CHARARA2 and Jean-Marc GIRAULT1 \\ ${ }^{1}$ Université François Rabelais de Tours, UMR « Imagerie \& Cerveau » Inserm U930, Tours, France \\ ${ }^{2}$ Department of Physics and Electronics, Faculty of Sciences I, Lebanese University, Beirut, Lebanon \\ ${ }^{3}$ ATYS Medical, Soucieu en Jarrest, France \\ ${ }^{4} \mathrm{CHU}$ de Lille, Lille, France
}

\begin{abstract}
Detection of small emboli, precursors of Cerebrovascular Accidents, is a worldwide concern since CVAs represent the second cause of mortality in the world. Computerized analysis of Transcranial Doppler signals can aid early detection of circulating emboli and micro-emboli. Commercially used systems of automatic emboli detection rely on standard short time Fourier transform techniques in which detection is based on constant thresholds. These standard algorithms do not offer robust detections and are incapable of detecting the smallest micro-emboli. To enhance this detection, we propose in this study optimized techniques based on novel methods of threshold application. By implementing our new time-varying threshold of detection, we were able to decrease the probability of non-detection and the probability of false alarm by around half the values obtained by standard techniques. Moreover, our new techniques were clearly efficient in exploiting the transient-like embolic signals and hence make detection of micro-emboli easier and more evident. This was proved by enhancing important parameters of which are the embolus to blood ratio and the peak to threshold ratio. Applied on our set of recorded signals, the new detectors allowed obtaining embolus to blood ratios twice greater than the embolus to blood ratios achieved by standard techniques and a sufficient increase in peak to threshold ratios.
\end{abstract}

Keywords-emboli detection; transcranial Doppler; short time Fourier transform; constant threshold; time-varying threshold

\section{Introduction}

Cerebrovascular Accidents (CVAs) are the second cause of mortality and morbidity worldwide [1]. CVAs are responsible for over 32,000 deaths per year in France and over 130,000 deaths in the United States of America. Thus CVAs represent a concern over a huge population.

Up to $14 \%$ of all strokes are caused by cerebral embolism [1]. Cerebral embolism is the blockage of blood flow in a cerebral artery by an embolus. An embolus may be a gas bubble, fat globule, blood clot or any other strange body carried by blood flow.

Transcranial Doppler (TCD) ultrasound is the most common system for the detection of asymptomatic emboli and micro-emboli circulating in the cerebral vascular system [1]. Micro-embolic events are detected in the TCD signal as high intensity transient signals superimposed on the Doppler signal backscattered by the blood. Doctors and clinicians worldwide are now firmly convinced that the detection of small microemboli is a precursor of the coming large embolus with more dangerous effects. Consequently, the precise and automatic detection of the smallest asymptomatic micro-emboli is an important challenge in support of CVA avoidance and prevention.

Audible detection alongside visualization of the spectrogram on the TCD system is considered as the gold standard of embolus detection. From a signal processing viewpoint, most of the commercial systems are based on Short Time Fourier Transform techniques employing constant thresholds of detection. While such systems perform well for relatively intense embolic signals, their performance in the detection of lower intensity micro-embolic signals is unsatisfactory [2-3].

In this work, we incorporated STFT method based on a novel thresholding technique where we tend to construct a threshold from the negative part of the Doppler signal. The underlying idea is that by using this type of thresholds, we exploit micro-events appearing in the positive part of the signal. Moreover, using this technique we amplify the embolus Doppler energy relative to the background Doppler energy. This new detection should allow the detection of the smallest micro-emboli and improve the accuracy of detection.

\section{Methods}

\section{A. Explanation of dataset}

TCD is a non-invasive, non-ionizing, inexpensive, portable and safe technique, which renders it as a convenient tool for the detection of cerebral micro-emboli. The TCD system we used to obtain our recordings, was the transcranial Holter (TCD-X, Atys Medical, Soucieu en Jarrest, France) shown in Fig. 2. By means of a transducer, an US wave with frequency of $1.5 \mathrm{MHz}$ was transmitted to the middle cerebral artery of patients with carotid stenosis. The pulse repetition frequency (PRF) was $6.4 \mathrm{kHz}$ and the US power was $50 \mathrm{~mW} / \mathrm{cm}^{-2}$.

During post-treatment, the TCD recordings obtained were converted into audio-wave files. These recordings were then analyzed and treated with our developed algorithms. 


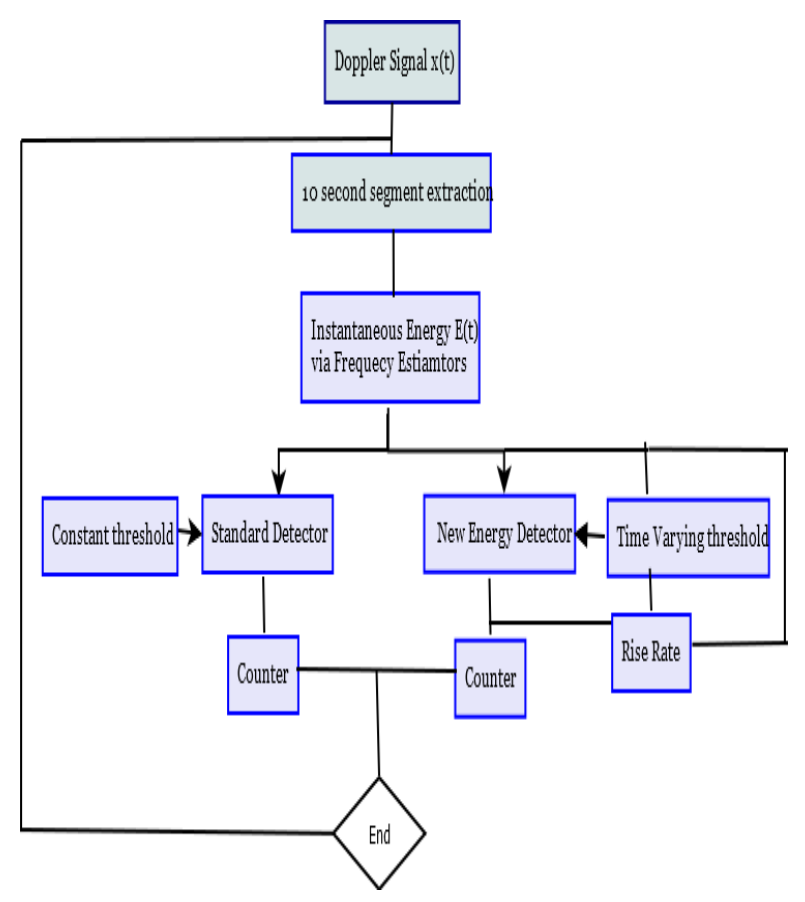

Fig. 1. A typical embolus detection system including standard detection and our new detection procedure

Primarily, the audio files were played and visualized in order to constitute our gold standard of detection. Embolic signals were identified on the basis of their characteristic sound and from visual inspection of the spectrogram. The positions in time of embolic and micro-embolic events were noted. Genuinely, listening to the audio files was made at both the normal speed and a second time at half of the normal speed. The latter process allowed detecting several microemboli that were previously inaudible.

\section{B. The Short Time Fourier Transform}

The most familiar of methods employed by commercial TCD ultrasound systems are based on the Short Time Fourier Transform. The Short Time Fourier Transform is an adapted form of the Fourier transform that analyzes only a small segment of the signal at a time -a technique called windowing of the signal or also Windowed Fourier Transform (WFT). This assures stationary of the signal within the analyzing window. The STFT employs a time-frequency representation known as the spectrogram. The spectrogram will be demonstrated in the next section.

\section{Energy Estimators}

It is widely stated that the energy of the backscattered Doppler signal is proportional to the size of the scattterer to the power of 6 [3]. Energy returned by an embolus is greater than that returned by billions of red blood cells. Hence, energy is able to provide the best information from which the presence of micro-emboli could be detected. This justifies why we chose our detectors to be majorly based on energy criteria.

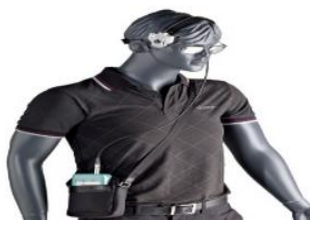

Fig. 2. Transcranial Doppler system (TCD-X, Atys Medical, Soucieu en Jarrest, France)

Two kinds of energy estimators can be used to assess the instantaneous energy of Doppler signals: those based on time estimators and those based on spectral or frequency estimators. The two types are short-term estimators and the calculation is carried out using a sliding window.

We will discuss spectral estimators since STFT or the spectrogram is based on these estimators. The frequency estimator with a sliding window can be formally written as:

$$
E(t)=\int\left|\int x(\tau) w^{*}(t-\tau) e^{-j w \tau} d \tau\right|^{2} d f
$$

$\mathrm{x}(\mathrm{t})$ is the analyzed Doppler signal, $\mathrm{w}(\mathrm{t})$ is a sliding window (covering 80\%) and * stands for complex conjugation. We carried out the calculation of the instantaneous energy on repetitively 10 second segments extracted from the Doppler signal.

\section{Energy detector with constant threshold: standard method}

After a stage of experimental optimization of the STFT parameters, the STFT in this study was performed using a 64 point Hamming window with an overlap of $65 \%$.

Micro-embolus signals are detected by a binary test used in all methods of detection.

$$
\begin{gathered}
\boldsymbol{H}_{1} \\
\stackrel{\boldsymbol{H}_{0}}{<}
\end{gathered}
$$

Decision information (DI) is a quantity generated, over a short duration, from the Doppler signal which contains the embolus signature. If the decision information is greater than the detection threshold then an embolus is detected (hypothesis H1), otherwise no embolus is detected (hypothesis $\mathrm{H} 0$ ). As we previously clarified, in current commercial devices the Decision Information on which detection is based is the instantaneous energy.

The choice of the threshold is a very crucial step before the identification of embolic signals and in the whole detection process. Although the instantaneous energy is time-varying, in standard methods, this energy is compared to a constant threshold. This constant threshold is empirical; i.e. it is fixed empirically by the user for the entire examination. This threshold is patient-, operator-, and device-dependent. The threshold is set above the maximal detected energy of the Doppler signal when no embolus is present, where this maximum energy always happens during the systole phase. Generally, for manual detection, the threshold is set $3 \mathrm{~dB}$ above this energy. For automatic detection, it is usually set between 3 to $9 \mathrm{~dB}$ above the highest energy and depends on the TCD system calibration [4]. Since the setting of this 
threshold is not the same for different examinations, comparisons between various applied methods are hard to achieve.

These empirical thresholds of detection have proved to be inefficient in many detection algorithms. In fact, these thresholds are adjusted too high, in a manner where microembolic signals of lower magnitude than that of the blood Doppler signal (mainly located at the systolic phase) are never detected. Indeed, in many clinical examinations the medical expert observes micro-emboli not detected by the system. This has led our team to achieve the detection with another threshold approach.

\section{E. Energy detector with a time-varying threshold}

We established a threshold that could help detect transient events in a robust manner. A threshold able to differentiate High Intensity Transient Signals (HITS) from general background noise and from spontaneous intensity fluctuations of physiologic Doppler signals. The solution we proposed was to obtain a threshold from the negative part of the signal being treated. The idea is based on the fact that emboli appear in the signal's positive part only, as will be demonstrated next. This threshold would be perfect in displaying the smallest embolic events occurring in the positive part.

After a smoothing step is applied (Fig. 3 a)), the trend of the energy signal is removed by subtracting this energy signal from its smoothed version (Fig. 3 b)). Knowing that the energy follows a Gaussian law [5], the resulting energy fluctuation can be represented by the following equation:

$$
E(t)=\mu(t)+\gamma(t)
$$

Where $\mu(\mathrm{t})$, the mean, represents the low variations of the energy and $\gamma(t)$, standard deviation, the high variations. As embolic signals are characterized by high variations [6], we tend to eliminate the term $\mu(\mathrm{t})$. The energy now is a centered energy denoted by $\gamma$. On the other hand, $\gamma(t)$ is a summation of the contribution of the positive and negative energy fluctuations. It is expressed as follows:

$$
\gamma(t)=\gamma(t)_{p o s}+\gamma(t)_{n e g}
$$

Moreover, the probability density of this energy, following a Gaussian distribution [5] is written in the following form:

$$
\boldsymbol{P}_{\gamma}(t)=\frac{1}{\sigma \sqrt{2 \pi}} e^{-\left(\frac{\gamma(t)}{2 \sigma^{2}}\right)^{2}}
$$

This probability density is also a summation of the probability densities from the positive and negative parts of the energy.

$$
\boldsymbol{P}_{\gamma}(t)=\boldsymbol{P}_{p o s}(t)+\boldsymbol{P}_{\text {neg }}(t)
$$

From our own experience and knowledge, embolic signals only appear in the positive part of the energy fluctuations. That means, applying the Gaussian statistics to the negative energy fluctuations, the probability of finding events outside the Gaussian statistics is zero. Applying the same statistics on the positive fluctuations, the latter probability is non zero. In other words, the latter statement could be represented by:

$$
P_{p o s}(t)=P_{n e g}(t)
$$

when no embolic signature is present and vice versa.

Hence, the positive part of the energy represents the same fluctuations as the negative part but corrupted with High Intensity Transient Signals (HITS) that are out of the Gaussian statistics. In other words, the positive part of the energy is symmetric to the negative one except when HITS occur.

In addition to the previous demonstrated facts, we know that the Doppler signal is cyclostationary [5]; its statistical properties vary cyclically with time. Therefore, we can hypothesize that the instantaneous energy is statistically periodic. From this cyclic energetic information an "instantaneous" threshold has been computed. This periodically time-varying threshold was computed from the negative energy fluctuations (Fig. 3 c)) by means of Hilbert transform and by finding the maximums of the Hilbert transform over small intervals (Fig. 3 d)). It was then applied onto the positive energy (Fig. $3 \mathrm{~b}$ ) black curve).

It could be clearly seen how, by using our methods, the peak values are clearly exposed due to the smoothing and thresholding steps. The time-varying threshold ensures precise and robust detection. Thus more meaningful results are obtained.

Peaks above the threshold are considered as suspicious micro-emboli and are extracted for further analysis. We will tend to refer to these peaks as High Intensity Transient Signals (HITS).

\section{1) Further analysis of the peaks detected above the} threshold.

The first step in the next analysis of the detected peaks is to differentiate those that denote suspicious embolic events from other peak values that may mislead the system. These peaks could be referred to as non-useful signals or unwanted peaks. They could be produced due to the transform method used. In fact, the STFT could give output values with ripples and noise-like small values. They may also represent normal fluctuations of the signal itself.

So in order to suppress these unwanted effects and hence improve our detectors reliability, we were able to establish a method of discrimination between these unwanted peaks and other important HITS based on the rise rate of each of the detected events. The rise rates were calculated as the difference between the time of onset of the peaks and the time at peak intensity. We noticed peaks having rise rate values that are in accordance with the prior information we primarily gathered on the emboli detected by the standard techniques and reported in our gold standard. Due to this observation, we decided to extract the HITS that correspond to the latter rise rate values and base our detection process on them.

\section{Results and discussion}

Fig. 4. Application of our detection technique 
a) Energy and the Smoothed version

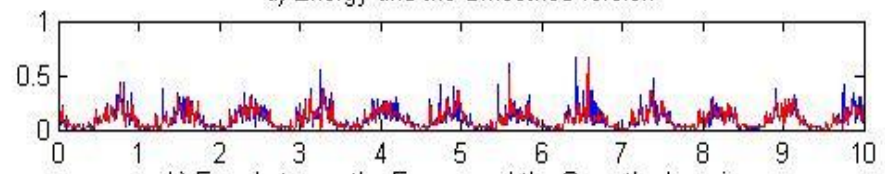

b) Error between the Energy and the Smoothed version
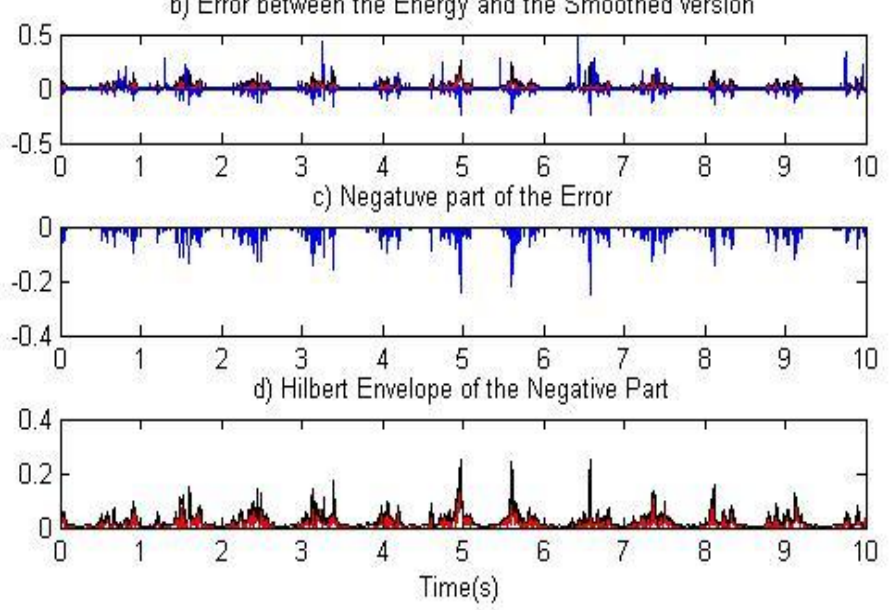

To evaluate the results of the different detectors, we compared these results to the gold standard of detection established initially. The basic statistical tools calculated for our evaluation were:

1) Probability of Non Detection $(P N D)=$ Number of Non detections (NND) / total Number of Detections (ND)

2) Probability of Non Detection $(P N D)=$ Number of Non detections (NND) / total Number of Detections (ND)

3) Probability of False Alarm $(P F A)=$ Number of False Alarm (NFA) / total Number of Detections (ND)

NND is the instance of not detecting an embolus which did pass the blood vessel sample volume (embolus marked in the gold standard) and can be referred to as false negative detection. NFA is the instance of detecting an embolus where in reality no embolus crossed and can be referred to as false positive detection. ND is the total number of detections.

Another comparison tool used to assess our results is the Embolus to Blood Ratio (EBR). EBR is the most widely used parameter in embolic signal detection. EBR indicates how strong an embolic signal is relative to the background Doppler signal. It can be calculated in the following manner:

$$
E B R=10 \log _{10}\left(\frac{P_{E+B}}{P_{B}}\right)
$$

$P_{E+B}$ is the backscattered power measured when an embolus and the blood are present in the Doppler sample volume. $P_{B}$ is the backscattered power measured from blood alone in the sample volume.

The PND and the PFA of the new detector were $16.60 \%$ $22.19 \%$ respectively. The PND and the PFA of the standard detector were $34.47 \%$ and $42.13 \%$ respectively. Hence, our new detector allowed the reduction of the PND and the PFA by approximately half of the standard values. Consequently, we affirm that a detector based on a time-varying threshold is more reliable and adapted than standard techniques.
TABLE I. Comparison of the PND, PFA, and EBR results for STFT with both constant and time-varying thresholds.

\begin{tabular}{|llll|} 
& PND(\%) & PFA(\%) & EBR(dB) \\
\hline $\begin{array}{l}\text { STFT } \\
\text { constant } \\
\text { threshold }\end{array}$ & 34.47 & 42.13 & 16.78 \\
\hline $\begin{array}{l}\text { STFT time- } \\
\text { varying } \\
\text { threshold }\end{array}$ & 16.60 & 22.19 & 34.30 \\
\hline
\end{tabular}

Moreover, the calculated EBRs for the two techniques showed values of $16.78 \mathrm{~dB}$ for standard detectors compared to $34.30 \mathrm{~dB}$ for detectors based on the time-varying threshold. The EBR was increased by more than the double. The decent performance of our detectors in terms of EBR demonstrates that such detectors are well adapted for the precise detection of the smallest emboli.

\section{Conclusion}

In this study, we propose a Short Time Fourier Transform technique combined with the use of a time-varying threshold as a tool for robust micro-embolus detection. The technique was compared to the classical Short Time Fourier Transform technique based on constant threshold. The results showed that using time-varying thresholds allowed better micro-embolic events detection.

The results confirm that the proposed method reveals embolic signatures more apparently. It allows increasing the EBR and thus is in consistency with the widely known agreement that any processing method that improves the EBR would be able to ameliorate the performance of an automated embolus detection system [6]. In addition, the decrease in the number of non-detections and in the number of false positive detections also emphasizes the efficiency of the time-varying threshold as a powerful method for embolus detection.

\section{REFERENCES}

[1] "The World Health Organization," The World Health Report,WHO, Geneva, 2000.

[2] N. Aydin and H. MArkus, "Detection of Embolic Signals Using Wavelet Transform," in International Workshop on Nonlinear Signals and Image Processing, Bucharest, 1999.

[3] J.-M. Girault and Z. Zhao, "Synchronous detector as a new paradigm for automatic microembolus detection," Int. J. Biomedical Engineering and Technology, vol. 14, no. 1, pp. 60-70, 2014.

[4] J. Girault, Biard, D. Kouame, A. Bleuzen and F. Tranquart, "Spectral Correlation of the embolic blood Doppler signal," in Acoustics, Speech and Signal Processing, Toulouse, 2006.

[5] J.-M. Girault, "Cerebral Microembolism Synchronous Detection with Wavelet Packets," in Signal and Image Multiresolution Analysis, New Jersey, John Wiley \& Sons, 2012.

[6] D. H. Evans, "Ultrasonic Detection of Cerebral Emboli," Ultrasonics, vol. 1 , no. 1 , pp. 316-326, 2003 